\title{
Experiments on Classification of Electroencephalography (EEG) Signals in Imagination of Direction using Stacked Autoencoder
}

\author{
Kenta Tomonaga, Takuya Hayakawa, Jun Kobayas hi \\ Department of Systems Design and Informatics, Kyushu Institute of Technology, \\ Kawazu 680-4, Iizuka, 820-8502, Japan \\ E-mail:jkoba@ces.kyutech.ac.jp \\ lab.jkoba.net
}

\begin{abstract}
This paper presents classification methods for electroencephalography (EEG) signals in imagination of direction measured by a portable EEG headset. In the authors' previous studies, principal component analysis extracted significant features from EEG signals to construct neural network classifiers. To improve the performance, the authors have implemented a Stacked Autoencoder (SAE) for the classification. The SAE carries out feature extraction and classification in a form of multi-layered neural network. Experimental results showed that the SAE outperformed the previous classifiers.
\end{abstract}

Keywords: electroencephalography, stacked autoencoder, neural network, portable EEG headset, imagination of direction

\section{Introduction}

Electroencephalography (EEG) is a non-invasive way for measuring human brain activity. A lot of studies on Brain Computer Interface (BCI) have been making use of EEG because of its greater availability than invasive ways. In addition, portable and low-cost EEG devices have been developed and readily accessible nowadays. There are, however, unclear points in the accuracy of those portable EEG devices. ${ }^{1,2}$ Therefore, the potential of applications using the devices should be explored.

Seto et al. studied on classification of EEG signals in imagination of direction measured by a medical EEG device. ${ }^{3}$ Following their study, we have employed a portable EEG headset to record EEG signals in imagination of direction, and implemented feature extraction with Principal Component Analysis (PCA) and several neural networks for the classification. ${ }^{4,5} \mathrm{We}$ validated the performance and confirmed that the best classification rate of the method using the medical EEG device was still better than those of our methods.

To achieve higher classification rate, we have implemented a Stacked Autoencoder (SAE) for feature extraction and classification of EEG signals in imagination of direction measured by the portable EEG device. G. E. Hinton et al. said that deep autoencoder networks can reduce the dimensionality of data much better than PCA. ${ }^{6}$ Therefore, we introduced a SAE to our study on classification of EEG signals in imagination of direction. Here we describe the implemented SAE and show results of comparative experiments that validate its effectiveness. 


\section{EEG Data Acquisition and Preprocessing}

Fig. 1 shows a wireless portable EEG headset developed by Emotiv Inc., named EPOC. ${ }^{7}$ We used the headset for EEG data acquisition in our preceding studies. ${ }^{4,5}$ EPOC has 14 electrodes and two reference electrodes, recording EEG signals at a sampling rate of $128 \mathrm{~Hz}$. The electrodes are placed on the scalp according to an extended 10-20 system for EEG measurement as shown in Fig. 2.

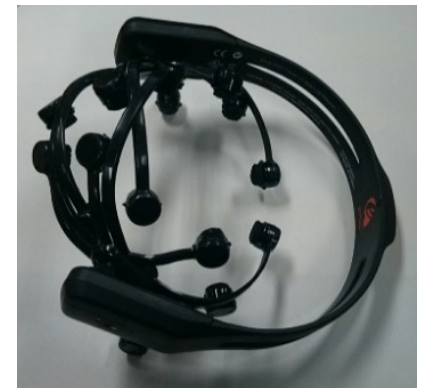

Fig. 1. Emotiv EPOC (wireless portable EEG headset)

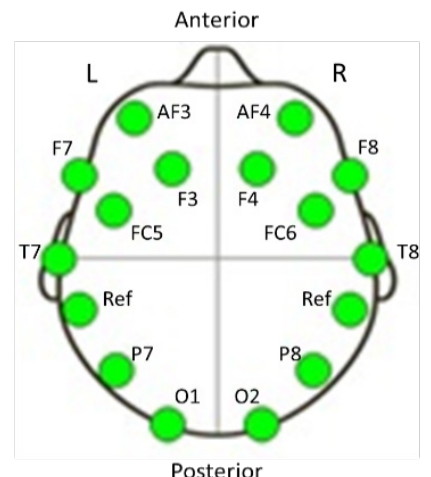

Fig. 2. Electrode placement of EPOC

Nine male university students participated in experiments as subjects for EEG data acquisition. Their average age was 21.9 years. Fig. 3 shows the experimental environment. During the experiments, a subject imagined one figure of arrows shown in Fig. 4. The obtained EEG signals were preprocessed to produce input vectors to a classifier. Fig. 5 is a flowchart of the preprocessing. The input vectors are composed of 23 elements. See Refs. 4 and 5 for more details about the EEG data acquisition and preprocessing.

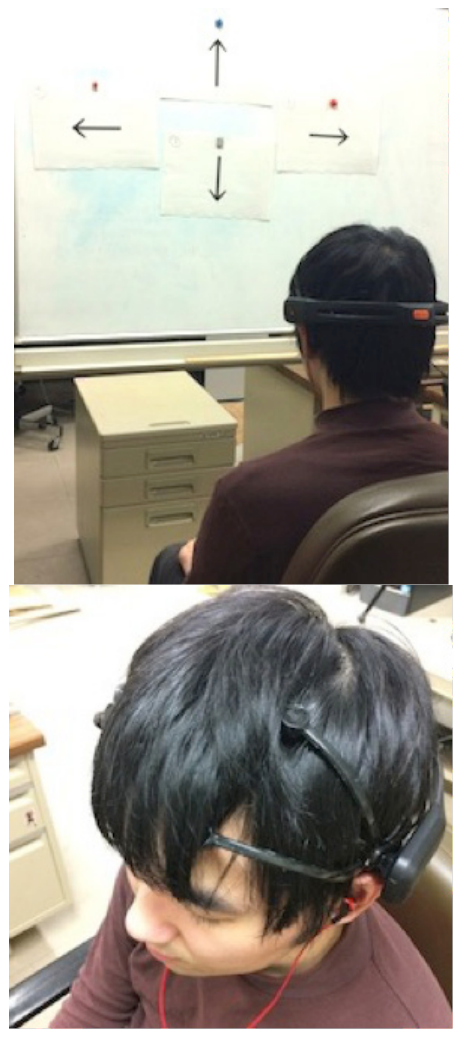

Fig. 3. Experimental environment

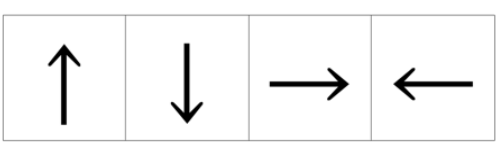

Fig. 4. Arrows indicating directions (up, down, right, and left)

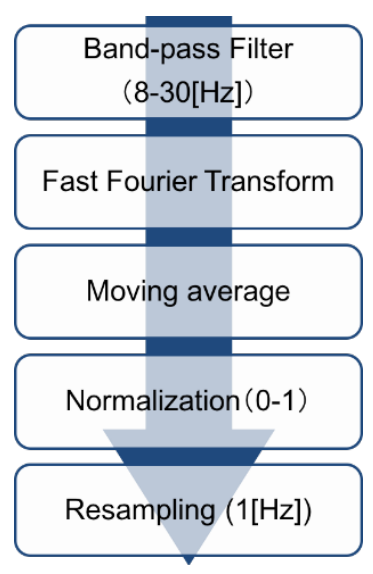

Fig. 5. Flowchart of preprocessing 


\section{Classification with Stacked Autoencoder}

In our previous studies, ${ }^{4,5}$ we applied PCA to the preprocessed EEG data for reducing the dimension, and then trained three-layered neural networks by means of backpropagation for 1000 or 10000 epochs with the dimensionally-reduced data as feature vectors and target values representing the direction a subject imagined. This classification method is called "PCA-NN" in this paper.

We have introduced deep neural networks in order to achieve better classification performance than the PCA-NN. Although training a deep neural network was difficult for backpropagation due to vanishing gradient problem, pretraining weights between nodes of a deep neural network can be a solution to the problem.

Stacked Autoencoder (SAE) is a way of constructing a deep neural network, in which deep architectures are initialized by stacking pretrained autoencoders. Fig. 6 illustrates a typical autoencoder that is an hourglass-shaped three-layered neural network. This neural network has the same number of nodes in the input and output layers, and the network is trained by backpropagation so that it can yield output values equal to given input ones. Autoencoder is an unsupervised learning method, which can be used for dimensionality reduction as mentioned above. The anterior part between the input and hidden layers of an autoencoder works as encoder, compressing input signals and extracting significant information from them. In SAE, the encoder parts of pretrained autoencoders are stacked for initializing a deep neural network.
Fig. 7 depicts the configuration of the SAE with two hidden layers adopted in this study. The hidden layers were pretrained in the way of learning autoencoder. The first thing we conducted was to train an autoencoder with the preprocessed EEG signals for 1000 epochs so that it could output signals identical to the input ones. The encoder part of the trained autoencoder was utilized as the pretrained first hidden layer of the SAE shown in Fig. 7, which generates signals from the EEG ones by reduction of dimensionality. Subsequently, we obtained by backpropagation for 1000 epochs another autoencoder producing signals further extracted from the output of the pretrained first hidden layer. The encoder part of the second autoencoder was set up as the pretrained second hidden layer in the SAE.

As stated above, the SAE in an initial state was composed of the pretrained encoders as the hidden layers. In addition, an output layer having four nodes representing the four directions was added to the network. The activation function of the output layer was softmax function, while Rectified Linear Unit (ReLU) for the hidden layers. The input layer had 23 nodes because input vectors to the SAE was made up of 23 elements produced from the preprocessed EEG signals. The number of nodes of the first and second hidden layers were tentatively set to 20 and 10 , respectively. Searching the optimum number of hidden layers and their nodes is a future work.

Finally, in order to achieve EEG signal classifiers, we conducted supervised learning, fine-tuning the initial SAE for 1000 or 10000 epochs using the preprocessed EEG signals with target values in one-hot representation indicating the imagined direction.

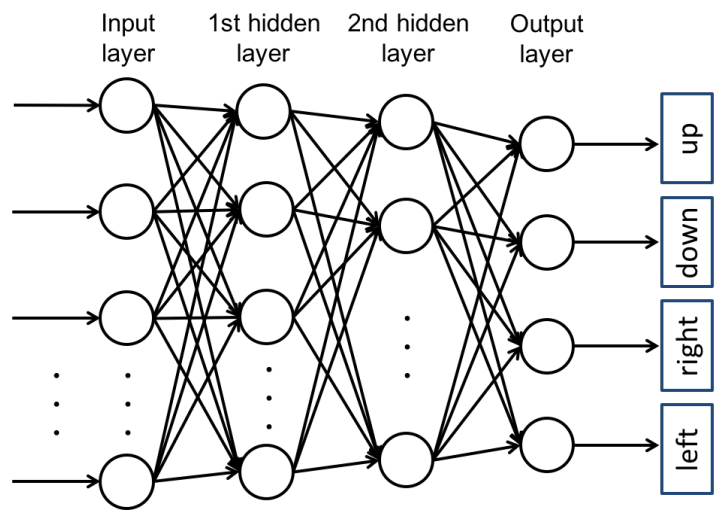

Fig. 7. Stacked Autoencoder with two hidden layers 


\section{Results and Discussion}

The classification rates of the classifiers were evaluated with 5 -fold cross validation. Table 1 and Table 2 show the evaluation results for the EEG signals obtained from one of the subjects. In the PCA-NNs, the PCA kept features with a $90 \%$ cumulative contribution ratio, and trimmed off the others. The PCA process resulted in producing 17-dimensional feature vectors. Therefore, the NNs of the PCA-NN were composed of 17-17-4 nodes in the input, hidden, and output layers. On the other hand, the structure of the SAEs were 23-20-10-4; the number of the input nodes was 23 that is equal to the dimension of the preprocessed input vector.

As shown in Table 1, the maximum classification rate by the PCA-NNs was $35.0 \%$ at FC5 electrode. It appears that overfitting caused the poor performance in some of the PCA-NNs trained for 10000 epochs.

Table 2 shows the classification rates of the SAEs. It clarified that the SAEs achieved better classification performance than the PCA-NNs. One of the SAEs realized $61.7 \%$ classification rate at FC5 electrode. Nevertheless, the work by Seto et al. using a medical EEG device ${ }^{3}$ is still better than the results obtained in this study.

It would be expected for improvement that a deeper SAE could provide superior performance than the SAEs with only two hidden layer used in this study. In addition, we will use denoising autoencoders ${ }^{8,9}$ to extract more relevant features for the classification of EEG signals.

Table 1. Classification rate percentages of PCA-NN

\begin{tabular}{ccc}
\hline Electrode & Epoch 1000 & Epoch 10000 \\
\hline AF3 & 25.8 & 25.8 \\
F7 & 29.2 & 26.7 \\
F3 & 26.7 & 29.2 \\
FC5 & 35.0 & 25.8 \\
T7 & 33.3 & 26.7 \\
P7 & 31.7 & 27.5 \\
O1 & 25.0 & 25.8 \\
O2 & 25.0 & 25.0 \\
P8 & 34.2 & 28.3 \\
T8 & 30.0 & 25.8 \\
FC6 & 25.8 & 26.7 \\
F4 & 27.5 & 24.2 \\
F8 & 24.2 & 24.2 \\
AF4 & 26.7 & 23.3 \\
\hline
\end{tabular}

Table 2. Classification rate percentages of SAE

\begin{tabular}{ccc}
\hline Electrode & Epoch 1000 & Epoch 10000 \\
\hline AF3 & 35.8 & 34.2 \\
F7 & 56.7 & 49.2 \\
F3 & 41.7 & 40.0 \\
FC5 & 55.8 & 61.7 \\
T7 & 40.8 & 40.8 \\
P7 & 37.5 & 41.7 \\
O1 & 30.0 & 33.3 \\
O2 & 28.3 & 30.8 \\
P8 & 24.2 & 29.2 \\
T8 & 45.0 & 32.5 \\
FC6 & 39.2 & 34.2 \\
F4 & 34.2 & 33.3 \\
F8 & 35.0 & 32.5 \\
AF4 & 36.7 & 35.0 \\
\hline
\end{tabular}

\section{Conclusion}

We implemented the SAEs for classification of EEG signals in imagination of direction, and compared the performance with those of the NNs trained using the feature vectors extracted by PCA. The results demonstrated that the SAEs achieved the improvement, however the achievement of the preceding study using a medical EEG device is still better than ours using the portable EEG headset. There remains much to explore a way to select the number of layers in the SAEs and to adopt denoising autoencoders as future work.

\section{References}

1. M. Duvinage et al., A P300-based quantitative comparison between the Emotiv Epoc headset and a medical EEG device, in Proc. of the $9^{\text {th }}$ IEEE/IASTED Int. Conf. on Biomedical Engineering (Innsbruck, Austria, 2012).

2. M. Duvinage et al., Performance of the Emotiv Epoc headset for P300-based applications, BioMedical Engineering On Line 12(56) (2013).

3. Y. Seto et al., Classification by EEG frequency distribution in imagination of directions, in Proc. of the 18th Int. Conf. on Knowledge-Based and Intelligent Information \& Engineering Systems (2014), pp. 1300 1306.

4. K. Tomonaga et al, Experiments on classification of electroencephalography (EEG) signals in imagination of direction using a wireless portable EEG headset, in Proc. of Int. Conf. on Control, Automation and Systems (Busan, South Korea, 2015), pp. 1805-1810. 
5. S. Wakamizu et al., Experiments on Neural Networks with Different Configurations for Electroencephalography (EEG) Signal Pattern Classifications in Imagination of Direction, in Proc. of IEEE Int. Conf. on Control System, Computing and Engineering (Penang, Malaysia, 2015), pp. 477-481.

6. G. E. Hinton and R. R. Salakhutdinov, Reducing the Dimensionality of Data with Neural Networks, Science 313 (2006), pp. 504-507.
7. Emotiv EPOC, https://emotiv.com/epoc.php.

8. P. Vincent et al., Extracting and Composing Robust Features with Denoising Autoencoders, in Proc. of the $25^{\text {th }}$ Int. Conf. on Machine Learning (Hels inki, Finland, 2008), pp. 1096-1103.

9. P. Vincent et al., Stacked Denoising Autoencoders: Learning Useful Representations in a Deep Network with a Local Denoising Criterion, J. of Machine Learning Research 11 (2010), pp. 3371-2408. 